\title{
The implementation of Korean adult's optimal formant setting by Praat scripting*
}

\author{
Jiyeon Park ${ }^{1} \cdot$ Cheoljae Seong ${ }^{2, * *}$

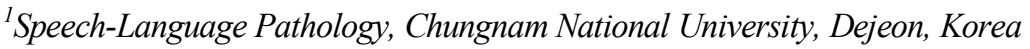 \\ ${ }^{2}$ Linguistics, Chungnam National University, Dejeon, Korea
}

\begin{abstract}
An automated Praat script was implemented to measure optimal formant frequencies for adults. Optimal formant analysis could be interpreted to show that the deviation of formant frequency that resulted from the two variously combined setting parameters (maximum formant and number of formants) was minimal. To increase the reliability of formant analysis, LPC order should be set differently, based on the gender or vowel type. Praat recommends $5,000 \mathrm{~Hz}$ and 5,500 Hz as maximum formant settings and, at the same time, recommends 5 as the number of formants for males and females. However, verification is needed to determine whether these recommended settings are valid for Korean vowels. Statistical analysis showed that formant frequencies significantly varied across the adapted scripts, especially with respect to the data on females. Formant plots and statistical results showed that linear_script and qtone_script are much more reliable in formant measurements. Among four kinds of scripts, the linear and qtone_scripts proved to be more stable and reliable. While the linear_script was designed to have a linearly increased formant step in for-loop, the increment of formant step in the qtone_script was arranged by quarter tone scale (base frequency $\times$ common ratio $(\sqrt[24]{2}))$. When looking at the tendency of the formant setting drawn by the two referred algorithms in the context of front vowel [i, e], the maximum formant was set higher; and the number of formants set at a lower value than recommended by Praat. The back vowel $[\mathrm{o}, \mathrm{u}]$, on the contrary, has a lower maximum formant and a higher number of formants than the standard setting.
\end{abstract}

Keywords: Praat script, optimal formant setting, Korean monopthong

\section{1. 서론}

모음은 성도 모양의 특성에 따라 달리 공명되어 산출되며 이 때 혀, 턱, 입술의 위치에 영향을 받는다. 모음을 효율적으로 분 석하기 위해서는 성도의 공명주파수로 정의되는 포먼트를 활
용할 수 있는데(Seong, 2005) 특히 첫 번째 공명주파수(first formant, 이하 F1)와 두 번째 공명주파수(second formant, 이하 F2)를 이용하여 조음과의 연관성을 밝혀낼 수 있다(Fry, 1982).

$\mathrm{F} 1$ 은 혀의 높이에 대한 조음 표지로 개구도에 비례하는 값이 며, F2는 혀의 전후 위치에 대한 조음 표지로 혀가 전방에 위치할

\footnotetext{
* 이 논문은 2019년 한국음성학회 봄 학술대회 발표논문을 수정, 보완한 것입니다.

** cjseong49@gmail.com, Corresponding author

Received 9 October 2019; Revised 19 November 2019; Accepted 21 November 2019

(c) Copyright 2019 Korean Society of Speech Sciences. This is an Open-Access article distributed under the terms of the Creative Commons Attribution NonCommercial License (http://creativecommons.org/licenses/by-nc/4.0) which permits unrestricted non-commercial use, distribution, and reproduction in any medium, provided the original work is properly cited.
} 
수록 그 값이 커진다(Behrman, 2007; Kent \& Read, 2002). 자음은 조음음성학적 측면에서 협착의 위치, 닫힘 정도, 유무성 여부 또는 조음 방법의 유형에 따라 특성을 정확히 기술할 수 있으나 모음은 혀, 턱, 입술의 위치에 의해 분석하기 때문에 자음과 비 교해서 특징을 기술하는 방법의 객관성이 떨어진다(Park, 2008). 이미 여러 선행연구에서 음향 분석이 말소리를 객관적으로 수 치화하고, 수치화된 말소리 특성이 음성의 병리적 진단과 치료 효과 판정에 사용될 수 있다고 설명한다(Lee, 2017; Jin, 2004). Song \& Seong(2018), Sim et al.(2016) 또한 같은 맥락에서 조음 치료 시 모음의 음향음성학적 변수인 모음 사각도, 모음 간 유 클리드 거리 등이 치료의 객관성을 확보할 수 있는 민감한 지표 로 활용될 수 있다고 설명하였다. 마비말 장애 화자에 적용된 포먼트 산점도 상의 FCR (formant centralization ratio)과 F2 ratio 는 비전형적인 음성 특성을 파악하고 치료 효과를 모니터링하 는 데 있어 중요한 변수라는 보고도 있다(Sapir et al., 2010). 따 라서 모음을 객관적 수치로 설명하기 위해 방법론적으로 타당 한 명시적인 포먼트 분석 방법을 제시할 필요가 있다.

포먼트 분석을 위해 사용되는 소프트웨어로는 CSL (Computerized Speech Lab), Dr. Speech (TigerDRS, USA), Praat (Boersma \& Weenink, 2014, Netherlands, 이하 '프랏'), WaveSurfer (KTH, Sweden) 등이 있다. 이들 소프트웨어 중 프랏은 포먼트 분석에 서 연구자의 요구와 모음 종류에 따라 다양한 변인을 고려한 정 교한 세팅을 할 수 있어서 큰 장점을 지니고 있다(Park \& Seong, 2018).

Escudero et al.(2009)은 프랏 측정 환경에서 최적의 포먼트 분 석은 최대 포먼트 주파수(maximum formant)와 포먼트 개수 (number of formants) 세팅에 따른 포먼트 측정치의 변이 (variance)가 최소일 때 이루어진다고 하였다. 이 견해를 기반으 로, Yoon \& Kang(2014)은 성인 한국어 단모음 데이터를 이용하 여 각 포먼트 값의 변이를 최소화하는 최적의 세팅으로 검출된 포먼트 값이 사람이 수동으로 측정한 값과 높은 상관관계를 가 짐을 밝혔다. 이러한 연구 맥락에서, 포먼트 세팅의 섬세한 변 경을 통해 좀 더 정확한 포먼트 값을 얻을 수 있다는 개연성이 확보된다.

프랏 매뉴얼에서는 성도의 크기 차를 반영하기 위해 최대 포 먼트 주파수를 성인 여성의 경우 $5,500 \mathrm{~Hz}$, 성인 남성의 경우 $5,000 \mathrm{~Hz}$ 로 설정할 것을 권고하였다(Boersma \& Weenink, 2014). 그러나 성인의 경우라도 후설 고모음/오, 우/의 경우는 분석 범 위를 설정하는 최대 포먼트 주파수 세팅값을 약간 낮추어야 더 정확한 값이 얻어진다는 관찰도 있다(Kim \& Seong, 2016). 후설 고모음은 조음 시 성도의 길이가 길어지면서 포먼트 주파수가 전반적으로 낮아지는 경향이 있는데 이러한 하강 경향을 기존 의 세팅으로 반영하기 어렵다. 전설 모음은 조음 시 좁힘점이 전설 부분에 형성되고 구강 내 F2 지배 공명강인 성도 수평면
(Horizontal plane)의 부피가 극단적으로 줄게 되면서 포먼트 세 팅을 정밀하게 고려하지 않으면 F1, F2 주파수의 정확한 측정이 어렵다(Fry, 1982). 특히 F2 주파수의 변동성이 커지는 경향이 있다. 최대 포먼트와 포먼트 개수를 정확히 짝지어 언급하지는 않았으나 Yang(2019)에서도 전설 모음의 포먼트 개수를 낮게 설정하여 분석을 실시하였다.

$\operatorname{Kim}(2015)$ 과 Song \& Seong(2018)에서는 아동의 모음 포먼트 를 다루면서 보다 정확한 포먼트 분석을 하고자 모음에 따라 포 먼트 개수와 최대 포먼트를 다르게 설정하였다. 이를 위해 수많 은 시행착오 과정을 거치는데 Park \& Seong(2018)에서는 이러 한 수작업이 필요한 시행착오 과정을 겪지 않고 최적의 아동 모 음 포먼트 분석을 하기 위한 목적으로 Praat의 최대 포먼트와 포 먼트 개수 자동 세팅 스크립트를 구현하였다.

기존의 많은 연구들이 성인의 포먼트 분석 시, 프랏에서 제공 하는 가이드로 최대 포먼트 설정 값을 남성 $5,000 \mathrm{~Hz}$, 여성 5,500 $\mathrm{Hz}$, 포먼트 측정 개수는 5 개를 따랐다. 그러나 이를 한국어 모음 에 적용했을 때도 타당한지 검증이 필요하다. 그리고 Yoon \& $\operatorname{Kang}(2014)$ 이 언급한 최소변이 세팅, Kim \& Seong(2016)에서 언급한 후설고모음의 특이성, $\operatorname{Yang}(2019)$ 에서 지적한 전설모음 의 특이성을 반영한 세팅 조건도 고려해야 한다. 따라서 본 연 구에서는 Park \& Seong(2018)에서 개발된 아동 포먼트 분석 스 크립트를 기반으로 성인 포먼트 분석에서 최적의 포먼트 세팅 을 찾도록 확장 구현하여 기존의 포먼트 분석 방법에 대한 타당 성을 검토하였다. 이 논문은 Park \& Seong(2018)의 후속 연구로 성인의 모음 포먼트 분석 모듈이 새롭게 고안되어 첨가되었다.

\section{2. 연구방법}

\section{1. 포먼트 세팅 스크립트 구현}

프랏에서 제시하는 LPC를 이용한 포먼트 분석 방법 중 Burg 방법이 F1과 F2를 검출하는데 가장 적합하다고 알려져 있다 (Childers, 1978; Press et al., 1992). 본 연구에서는 Window 10 환 경에서 프랏 ver. 5.3.141를 이용하여 모음 분석을 위한 최적의 포먼트 세팅 스크립트를 구현하였다.

서론에서 언급한 바와 같이 최대 포먼트와 포먼트 개수 두 가 지 조건을 반복문으로 설계하여 알고리듬이 설정한 모든 경우 의 수에서 $\mathrm{F} 1$ 측정치의 변이와 $\mathrm{F} 2$ 측정치의 변이의 합이 가장 작은 경우를 찾아 그 때 도출되는 최대 포먼트, 포먼트 개수를 최적의 포먼트 세팅 조건으로 간주하였다(Park \& Seong, 2018). 분석조건에 활용되는 한국어 모음은 7모음 체계(아, 에 이, 오, 우, 으, 어)를 채택하였다(Bae, 2003). 이를 위하여 알고리듬을 서로 다르게 설계한 4가지 방식의 스크립트로 접근하였다.

첫 번째 스크립트는 프랏 매뉴얼의 권고에 따랐다. 즉 모든 모음에 대하여 성별에 따라 최대 포먼트는 남성 $5,000 \mathrm{~Hz}$, 여성

1 본 연구는 프랏의 모든 버전에서 운용가능하도록 ver.5.3.14를 이용하여 스크립트를 설계하였다. 이는 프랏의 버전에 따라 코딩 규약이 다른 점을 고려한 것이다. 이에 대한 설명은 Park \& Seong(2018)에 기술하였다. 
$5,500 \mathrm{~Hz}$ 로 고정하며, 포먼트 개수는 두 성별 모두 5로 고정한 후 포먼트를 검출하였다(fixed_5000n5_script로 명명).

두 번째 스크립트는 최대 포먼트를 프랏의 권고에 따라 설정 한 후, 포먼트 개수에 변이를 주었다. 포먼트 개수에 변이를 주 는 것은 본 연구에서 사용하는 포먼트 분석 알고리듬이 포먼트 개수의 2 배 값을 갖는 LPC 차수를 이용하는 Burg 방법이기 때 문이다. 포먼트 개수는 4 부터 6 까지 0.5 단위로 증가하는 5 가지 경우의 수를 설정하였고, 최대 포먼트와 조합하여 최적의 세팅 값을 찾도록 설계하였다(fixed_script로 명명).

세 번째 스크립트는, 포먼트 개수가 4부터 6까지 0.5단위로 증가하는 5 가지의 경우의 수로 상위 for 반복문에 먼저 설계하 고 최대 포먼트는 $100 \mathrm{~Hz}$ 씩 증가하는 20 가지 경우의 수로 하위 for 반복문에 배치하였다. 결과적으로 하나의 모음에 대하여 총 $100(=20 \times 5)$ 가지 경우의 수에서 최적의 세팅 조건을 찾고 그에 상응한 포먼트 값을 출력한다(linear_script로 명명).

그리고 네 번째 스크립트에서는 청지각 척도인 반음척도 (semi-tone scale)의 절반에 해당하는 4 분음 척도(quarter-tone scale)를 적용하여 인간의 청지각적 특성을 고려한 스크립트를 구현하였다(Park \& Seong, 2018; Seong et al, 2008). 4분음 등비 수열을 적용하여 공비(common ratio)로 $(\sqrt[24]{2})$ 를 곱해가는 알고 리듬으로 포먼트 주파수는 총 14회 증가하면서 반복하며, 여기 에 포먼트 개수 5 가지를 조합하여 총 $70(=14 \times 5)$ 회의 반복조건 에서 최적의 세팅을 찾게끔 설계하였다(qtone_script로 명명).

각 스크립트별 최대 포먼트 및 포먼트 개수의 설정을 표 1 과 표 2에 정리하고, linear_script의 핵심 코드를 부록에 첨부하였다.

표 1. 스크립트별 최대 포먼트 설정 범위

Table 1. Setting range of maximum formant according to the scripts

\begin{tabular}{|c|c|c|}
\hline \multirow{2}{*}{ 스크립트 } & \multicolumn{2}{|c|}{ 최대 포먼트 설정 범위 } \\
\hline & 남 & 여 \\
\hline Fixed_5000n5_script & \multirow{2}{*}{5,000} & \multirow{2}{*}{5,500} \\
\hline Fixed_script & & \\
\hline $\begin{array}{c}\text { Linear_script }{ }^{2} \\
\text { (Loop } 100,100 \mathrm{~Hz} \text { 단위) }\end{array}$ & $4,000 \sim 5,900$ & $4,500 \sim 6,400$ \\
\hline $\begin{array}{c}\text { Qtone_script }{ }^{3} \\
\text { (Loop } 70, \sqrt[24]{2} \text { 공비 단위) }\end{array}$ & $4,000 \sim 6,000$ & $4,500 \sim 6,800$ \\
\hline
\end{tabular}

표 2. 스크립트별 포먼트 개수 설정 범위 Table 2. Number of formants according to the scripts

\begin{tabular}{c|c}
\hline 스크립트 & 포먼트 개수 설정 범위 \\
\hline Fixed_5000n5_script & 5 \\
\hline Fixed_script & $4 \sim 6$ \\
\hline Linear_script & $(0.5$ 단위 $)$ \\
\hline Qtone_script
\end{tabular}

2.2. 자료 수집

\subsection{1. 연구 대상 및 녹음 과제}

대전·충청 지역 15 년 이상 거주한 만 20 세에서 29 세 사이의 대학생 중 구강조음기관 및 음성에 이상이 없으며 정상 청력을 가진 성인 총 44명(남 16 명, 여 28명)을 대상으로 하였다. 녹음 과제는 한국어 7모음 체계 [아, 에, 이, 오, 우, 으, 어]로 구성하였 으며, \{모음+다\}의 형태로 [아다, 에다, 이다, 오다, 우다, 으다, 어다]를 3 회 발화하도록 하였다. 과제를 \{모음+다\}로 선정한 것 은 자연스럽지 못한 인위적인 운율을 배제하기 위해 문장틀에 넣어 발화하게 하기 위함이다(Seong, 2005). 종결어미 [-다]는 한 국어 동사의 기본형으로 자연스러운 단어를 만들고 동시에 마 지막으로 혀의 움직임을 화자마다 같게 하여 동일한 환경을 조 성하는 효과가 있다(Kim, 2012).

\subsection{2. 음성자료 처리}

음성 수집은 충남대학교 인문대학 음성음향실험실 내 방음 실에서 이루어졌다. Linear PCM 휴대용 녹음기(TASCAM DR-5, TEAK Corp., USA)로 녹음하였고, $44,100 \mathrm{~Hz}$ 추출률(sampling rate), 16 bit 양자화(quantization), 모노(mono) 조건에서 진행하 였으며 마이크는 내장된 콘덴서 마이크를 이용하였다. 녹음기 는 화자의 입으로부터 $15 \mathrm{~cm}$ 정도 간격을 두었으나 화자의 목 소리가 작다고 판단되는 경우 연구자가 거리를 조정하며 녹음 을 실시하였다. 총 3 회 녹음된 음성파일 중 안정적으로 녹음된 음성파일 1 회분을 분석대상으로 하였다. 각 모음 발성에서 안 정적인 펄스 신호가 유지되고, 강도(intensity)가 일정한 부분을 안정구간으로 추출하여 분석을 실시하였다.

\section{3. 통계}

통계 분석을 위해서 SPSS version 24 (IBM, USA)를 이용하였 다. 스크립트 간 최적의 포먼트 세팅(최대 포먼트, 포먼트 개수) 및 검출된 F1, F2에 대한 차이를 파악하기 위해 혼합 2 way 반복 측정 분산분석(mixed 2 way repeated measures ANOVA)를 실시 하였다. 개체 내 변수는 4개의 스크립트, 개체 간 변수는 7개의 모음으로 설정하였고 통계적으로 유의한 변수에 대해서는 정 밀한 해석을 위해 사후검정을 실시하였다. 사후 검정 시 다중비 교(multiple comparison)로 인해 나타나는 1종 오류의 증가를 조 정하기 위하여, Bonferroni alpha correction을 적용하여 결과를 해석하였다.

$2 \mathrm{Kim} \&$ Seong(2016)를 참고하여 후설고모음/오, 우/의 경우에 한해서 최대 포먼트 범위를 $500 \mathrm{~Hz}$ 하향 조정하여 남성은 3,500 5,400, 여성은 4,000 5,900 범위에서 최적의 세팅 조건을 찾도록 하였다.

3 qtone_script의 최대 포먼트는 linear_script의 최대 포먼트 초기값에 $\sqrt[24]{2}$ 를 14 승 하였을 때 계산되는 값이 포함되도록 범위를 설정하였다. 남성의 경우는 $4,000 \times(\sqrt[24]{2})^{14}=5,994$, 여성의 경우는 $4,500 \times(\sqrt[24]{2})^{14}=6,742$ 와 같이 계산된다. 


\section{3. 연구 결과}

3.1. 포먼트 세팅 결과 비교

\subsection{1. 포먼트 세팅 결과에 대한 기술통계}

Fixed_script, linear_script, qtone_script를 이용하여 모음별 최 적의 포먼트 설정으로 세팅된 최대 포먼트, 포먼트 개수의 중앙 값과 $\mathrm{IQR}$ 을 표 3에 정리하였다. 남성과 여성의 경우 모두 linear_script, qtone_script 결과에서 전설 모음의 최대 포먼트가 비교적 높고, 후설 모음의 최대 포먼트가 비교적 낮은 경향이 나타난 것을 알 수 있다. 또한 포먼트 개수는 전설모음에서 낮 고, 후설 모음에서 비교적 높은 경향을 확인할 수 있다.

\subsection{2. 최적의 포먼트 세팅에 대한 혼합 반복측정 분산분석}

최대 포먼트는 fixed_5000n5_script와 fixed_script가 동일하게 남녀 각각 $5,000 \mathrm{~Hz}, 5,500 \mathrm{~Hz}$ 로 고정하였으므로 fixed_5000n5 _script와 linear_script, qtone_script의 세팅 결과에 대해 검정을 실시하였다. 포먼트 개수에 대해서는 값을 5로 고정한 fixed 5000n5_script를 포함하여 변이를 설정했던 fixed_script, linear_ script, qtone_script까지 4개 스크립트 간 차이에 대해 검정하였다.

먼저 남성에 대한 검정 결과, 최대 포먼트는 스크립트 간 통 계적으로 유의한 차이가 있는 것으로 나타났으며 $[F(2,210)=$ $56.524, p<.001]$. 포먼트 개수 역시 스크립트 간 통계적으로 유의 한 차이가 있는 것으로 나타났다 $[F(2.635,276.685)=12.633, p$ $<.001]$. 또한, 최대 포먼트 $(p<.001)$ 와 포먼트 개수 $(p<.001)$ 모두 모음과 상호작용에서 유의한 차이가 있는 것으로 나타나 모음 간 세팅 결과에 차이가 있을 것을 확인할 수 있다. 이에 대해 Bonferroni 사후 검정을 실시하여 변수내 세부적인 대응 양상을 살펴보았다.

표 4는 남성 모음에서 스크립트 간 차이를 살펴본 결과다. 최 대 포먼트는 모음/아, 오, 우, 으/에서 fixed_5000n5_script와 linear_script, qtone_script 쌍에서 각각 유의한 차이가 있는 것을 알 수 있다. 또한 모음/오, 우/에서는 linear_script와 qtone_script
쌍에도 유의한 차이가 있는 것으로 나타났다. 모음 /이/에서는 fixed_5000n5_script와 qtone_script, linear_script와 qtone_script 쌍에서 스크립트 간 유의한 차이가 있는 것을 알 수 있다. 포먼 트 개수에서는 모음/에, 오/를 제외한 모든 모음에서 fixed 5000n5_script가 변이를 주었던 스크립트들과 유의한 차이를 보 이는 경우가 있는 것으로 나타났다.

표 3. 스크립트에 따른 모음별 포먼트 세팅 결과에 대한 기술통계 중앙값(IQR)

Table 3. Descriptive statistics for the formant settings for each vowel provided by 3 scripts_median (IQR)

\begin{tabular}{|c|c|c|c|c|c|c|c|c|c|}
\hline \multirow{2}{*}{\multicolumn{2}{|c|}{ 모음(세팅 변수) }} & \multicolumn{4}{|c|}{ 남성 } & \multicolumn{4}{|c|}{ 여성 } \\
\hline & & Fixed_5000n5 & Fixed & Linear & Qtone & Fixed_5000n5 & Fixed & Linear & Qtone \\
\hline \multirow{2}{*}{ 이 } & 최대 포먼트 & $5,000.0$ & $5,000.0$ & $4,300.0(800.0)$ & $4,365 \cdot 0(823$. & $5,500.0$ & $5,500.0$ & $5,350.0(975.0)$ & $359.0(1,285.0)$ \\
\hline & 포먼트 개수 & 5.0 & $5.5(0.6)$ & $5.5(0.625)$ & $5.5(0.8)$ & 5.0 & $4.5(1.0)$ & $5.0(1.1)$ & $5.0(1.1)$ \\
\hline \multirow{2}{*}{ 에 } & 최대 포먼트 & $5,000.0$ & $5,000.0$ & $4,950.0(1,275.0)$ & $4,695.5(1,057.8)$ & $5,500.0$ & $5,500.0$ & $450.0(1,125.0)$ & $5,763.5$ \\
\hline & 포먼트 개수 & 5.0 & $5.0(1.0)$ & 5.3( & 5.0 & 5.0 & 4.5 & 4.5 & \\
\hline \multirow{2}{*}{ 이 } & 최대 포먼트 &, 000.0 & $5,000.0$ & $4,450.0(875.0)$ & $4,494.0(840.5$ & $5,500.0$ & $5,500.0$ & $5,400.0(1,025.0)$ & $5,438.0(1,320.3$ \\
\hline & 포먼트 개수 & 5.0 & $5.0(1.0)$ & $4.5(0.3)$ & $4.5(0.5)$ & 5.0 & $4.5(1.0)$ & $4.5(1.0)$ & $4.5(0.8)$ \\
\hline \multirow{2}{*}{ 오 } & 최대 포먼트 & $5,000.0$ & $5,000.0$ & $4,250.0(650.0)$ & $4,365.0(581.8$ & $5,500.0$ & $5,500.0$ & $4,400.0(625.0)$ & $4,983.5(610.5)$ \\
\hline & 포먼트 개수 & 5.0 & $5.3(0.5)$ & $5.5(1.1)$ & & 5.0 & $5.5(1.0)$ & & \\
\hline \multirow{2}{*}{ 우 } & 최대 포먼트 & 000.0 & $5,000.0$ & $4,050.0(800.0)$ & $4,302.5($ & $5,500.0$ & $5,500.0$ & $4,400.0$ & $4,911.0(610.5)$ \\
\hline & 포먼트 개수 & 5.0 & 5.5( & & & & & & 5.8 \\
\hline \multirow{2}{*}{ 으 } & 최대 포먼트 & $5,000.0$ & $5,000.0$ & $4,150.0(325.0)$ & $4,118.0(397.3$ & $5,500.0$ & $5,500.0$ & $5,250.0(1,100.0)$ & $5,130.5(1,047.0)$ \\
\hline & 포먼트 개수 & 5.0 & $5.5(0.5)$ & $5.5(1.0)$ & $5.5(0.6)$ & 5.0 & $5.0(1.0)$ & $5.0(1.0)$ & $5.3(1.0)$ \\
\hline \multirow[t]{2}{*}{ 어 } & 최대 포먼트 & $5,000.0$ & $5,000.0$ & $4,350.0(1,000.0)$ & $4,365.0(1,112.0)$ & $5,500.0$ & $5,500.0$ & $5,150.0(1,250.0)$ & $4,983.5(958.0)$ \\
\hline & 포먼트 개수 & 5.0 & $5.5(0.6)$ & $5.5(1.0)$ & $5.5(1.0)$ & 5.0 & $5.0(0.5)$ & $5.0(1.1)$ & $5.0(1.5)$ \\
\hline
\end{tabular}


표 4. 모음에 따른 스크립트별 최대 포먼트 및 포먼트 개수에 대한 짝대응비교(남성)

Table 4. Pairwise comparisons for maximum formant and number of formants for each vowel with respect to the 4 kinds of scripts (male)

\begin{tabular}{|c|c|c|c|c|c|}
\hline $\begin{array}{l}\text { 세팅 } \\
\text { 변수 }\end{array}$ & $\begin{array}{l}\text { 모 } \\
\text { 음 }\end{array}$ & I & $\mathrm{J}$ & $\begin{array}{c}\text { 평균차이 } \\
(\mathrm{I}-\mathrm{J})\end{array}$ & Sig. \\
\hline \multirow{12}{*}{$\begin{array}{l}\text { 최대 } \\
\text { 포먼트 }\end{array}$} & \multirow{2}{*}{ 아 } & \multirow{2}{*}{ Fixed_5000n5 } & Linear & 500 & $0.003^{* *}$ \\
\hline & & & Qtone & 453.813 & $0.004^{* *}$ \\
\hline & \multirow{2}{*}{ 이 } & Fixed_5000n5 & Qtone & 47.438 & $0.040^{*}$ \\
\hline & & Linear & Qtone & -413.5 & $0.004^{* *}$ \\
\hline & \multirow{3}{*}{ 오 } & \multirow{2}{*}{ Fixed_5000n5 } & Linear & 793.750 & $0.000^{* *}$ \\
\hline & & & Qtone & 466.313 & $0.003^{* *}$ \\
\hline & & Linear & Qtone & $-327,438$ & $0.021^{*}$ \\
\hline & \multirow{3}{*}{ 우 } & \multirow{2}{*}{ Fixed_5000n5 } & Linear & 900 & $0.000^{* *}$ \\
\hline & & & Qtone & 486.5 & $0.002^{* *}$ \\
\hline & & Linear & Qtone & -413.5 & $0.002^{* *}$ \\
\hline & \multirow{2}{*}{ 으 } & \multirow{2}{*}{ Fixed_5000n5 } & Linear & 718.75 & $0.000^{* *}$ \\
\hline & & & Qtone & 733.5 & $0.000^{* *}$ \\
\hline \multirow{9}{*}{$\begin{array}{l}\text { 포먼트 } \\
\text { 개수 }\end{array}$} & 아 & Fixed_5000n5 & Fixed & -0.5 & $0.000^{* *}$ \\
\hline & \multirow{3}{*}{ 이 } & \multirow{2}{*}{ Fixed_5000n5 } & Linear & 0.469 & $0.025^{*}$ \\
\hline & & & Qtone & 0.531 & $0.004^{* *}$ \\
\hline & & Fixed & Qtone & 0.469 & $0.023^{*}$ \\
\hline & \multirow{2}{*}{ 우 } & \multirow{2}{*}{ Fixed_5000n5 } & Fixed & -0.594 & $0.000^{* *}$ \\
\hline & & & Qtone & -0.469 & $0.016^{*}$ \\
\hline & 으 & Fixed_5000n5 & Fixed & -0.625 & $0.000^{* *}$ \\
\hline & \multirow{2}{*}{ 어 } & \multirow{2}{*}{ Fixed_5000n5 } & Fixed & -0.438 & $0.001^{* *}$ \\
\hline & & & Qtone & -0.469 & $0.016^{*}$ \\
\hline
\end{tabular}

여성에 대한 검정 결과를 살펴보면, 최대 포먼트에 대해서 스 크립트 간 통계적으로 유의한 차이가 있는 것이 확인된다 [F(1.993 376.754)=43.652, $p<.001]$. 또한 모음과의 상호작용에서 유의한 차이가 있는 것으로 나타나 $[F(11.960,376.754)=7.482$, $p<.001]$ 모음 간 최대 포먼트 세팅의 차이가 있음을 알 수 있다. 포먼트 개수에 대해서는 스크립트 간 통계적으로 유의한 차이 가 없었으나 $(p=.213)$ 모음과의 상호작용에서 유의한 차이가 있 는 것으로 보아 모음에 따라 포먼트 개수 세팅에 차이가 있는 것을 알 수 있다 $[F(14.512,457.115)=2.986, p<.001]$.

표 5 는 여성 모음의 스크립트 간 차이를 살펴본 결과다. 최대 포먼트는 모음/오, 우/에서 fixed_5000n5_script, linear_script, qtone_script 사이에서 각각 유의한 차이가 나타난 것을 알 수 있 고, 모음/어/에서는 fixed_5000n5_script와 qtone_script 간 유의 한 차이가 있는 것으로 나타났다. 또한 포먼트 개수에서도 fixed_5000n5_script와 변이를 주었던 fixed_script, qtone _sript 사이에서 유의한 차이가 있는 것을 확인할 수 있다.
표 5. 모음에 따른 스크립트별 최대 포먼트 및 포먼트 개수에 대한 짝대응비교(여성)

Table 5. Pairwise comparisons for maximum formant and number of formants for each vowel with respect to the 4 kinds of scripts (female)

\begin{tabular}{|c|c|c|c|c|c|}
\hline $\begin{array}{l}\text { 세팅 } \\
\text { 변수 }\end{array}$ & $\begin{array}{l}\text { 모 } \\
\text { 음 } \\
\end{array}$ & I & $\mathrm{J}$ & $\begin{array}{c}\text { 평균차이 } \\
\text { (I-J) }\end{array}$ & Sig. \\
\hline \multirow{7}{*}{$\begin{array}{l}\text { 최대 } \\
\text { 포먼트 }\end{array}$} & \multirow{3}{*}{ 오 } & \multirow{2}{*}{ Fixed_5000n5 } & Linear & 1,025 & $0.000^{* *}$ \\
\hline & & & Qtone & 434.143 & $0.002^{* *}$ \\
\hline & & Linear & Qtone & -590.857 & $0.000^{* *}$ \\
\hline & \multirow{3}{*}{ 우 } & \multirow{2}{*}{ Fixed_5000n5 } & Linear & 928.571 & $0.000^{* *}$ \\
\hline & & & Qtone & 467.286 & $0.001^{* *}$ \\
\hline & & Linear & Qtone & -461.286 & $0.000^{* *}$ \\
\hline & 어 & Fixed_5000n5 & Qtone & 368.714 & $0.009^{* *}$ \\
\hline \multirow{7}{*}{$\begin{array}{l}\text { 포먼트 } \\
\text { 개수 }\end{array}$} & 아 & Fixed_5000n5 & Fixed & 0.321 & $0.017^{*}$ \\
\hline & 에 & Fixed_5000n5 & Fixed & 0.393 & $0.002^{* *}$ \\
\hline & 이 & Fixed_5000n5 & Fixed & 0.339 & $0.010^{*}$ \\
\hline & \multirow{2}{*}{ 오 } & \multirow{2}{*}{ Fixed_5000n5 } & Fixed & -0.464 & $0.000^{* *}$ \\
\hline & & & Qtone & -0.429 & $0.009^{* *}$ \\
\hline & \multirow{2}{*}{ 우 } & \multirow{2}{*}{ Fixed_5000n5 } & Fixed & -0.357 & $0.006^{* *}$ \\
\hline & & & Qtone & -0.482 & $0.002^{* *}$ \\
\hline
\end{tabular}

3.2. 스크립트별 포먼트 분포

\subsection{1. 남성의 스크립트별 포먼트 분포}

프랏의 권고 설정에 따른 fixed_5000n5_script를 이용하여 남 성의 각 모음별로 측정된 포먼트 분포를 산점도로 확인하였다 (그림 1). 편차 범위가 비교적 안정적인 다른 모음에 비하여 원 순 후설 고모음/우/에서 포먼트 편차 범위가 넓은 것을 확인할 수 있다. /우/의 편차 범위가 대각선 방향인 것은 F1, F2 모두 편 차가 크다는 것을 의미한다.

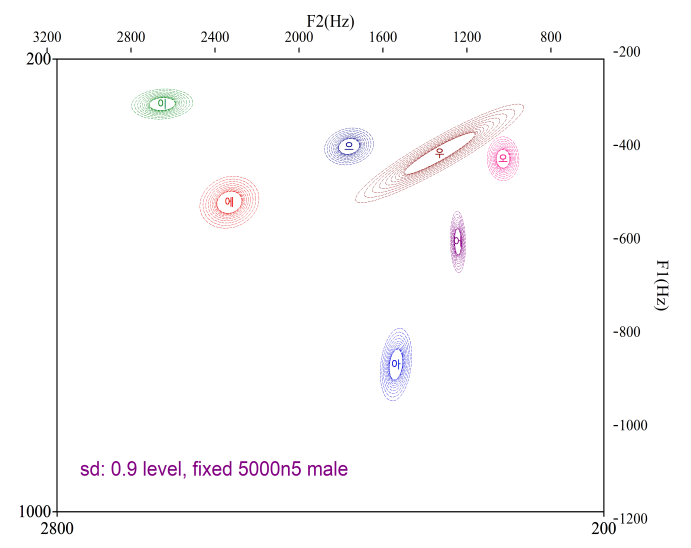

그림 1. fixed_5000n5_script를 이용한 포먼트 분포(남성) Figure 1. Formant distribution by fixed_5000n5_script (male)

그림 2는 포먼트 개수에만 변이를 설정했던 fixed_script를 이 용한 남성의 포먼트 분포다. 그림 1 과 비교하여 모음 /우/에 편 차 범위가 크게 감소한 것을 확인할 수 있으며, 7 개 모음의 각 영역이 뚜렷하게 구분되는 것을 알 수 있다. 


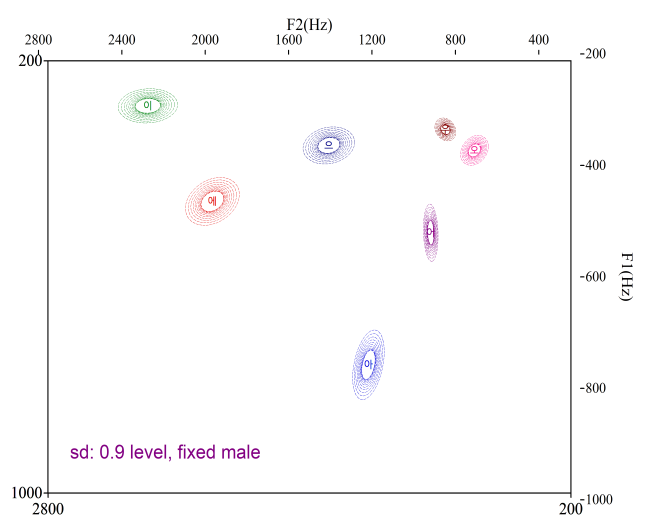

그림 2. fixed_script를 이용한 포먼트 분포(남성)

Figure 2. Formant distribution by fixed_script (male)

Linear_script, qtone_script를 이용한 포먼트 분포는 각 스크립 트에서 설정된 최적의 최대 포먼트, 포먼트 개수의 중앙값으로 포먼트를 측정한 후 얻은 것이다. 그림 3과 그림 4는 유사한 형 태를 보이며 각 모음의 영역이 적절히 분리된 것을 알 수 있다. 그림 1 과 비교했을 때, 모음/우, 어/의 편차가 감소된 것을 확인 할 수 있다.

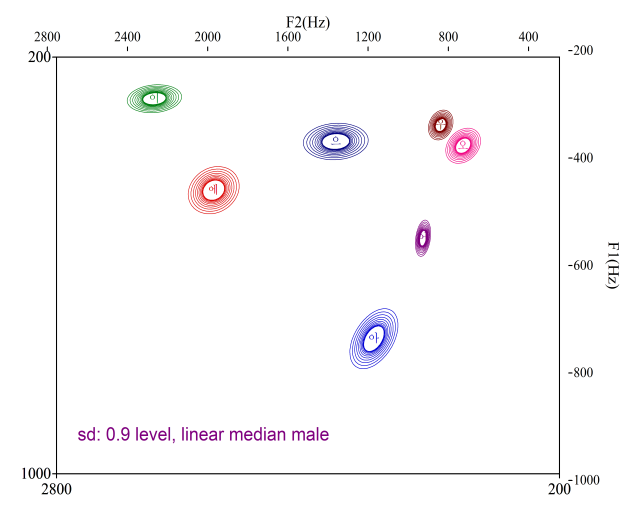

그림 3. Linear_script를 이용한 포먼트 분포(남성) Figure 3. Formant distribution by linear_script (male)

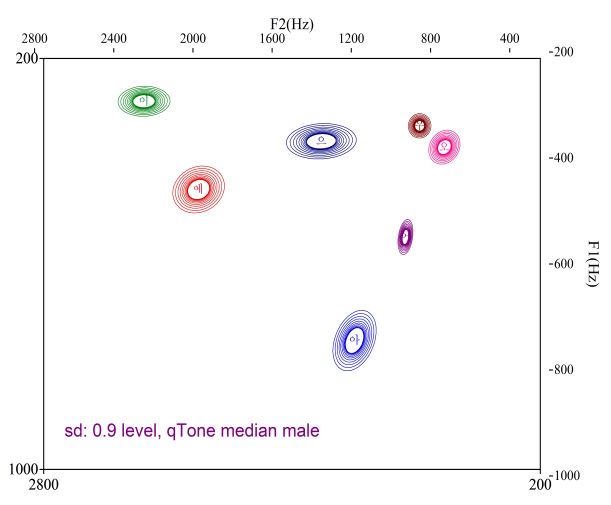

그림 4. qtone_script를 이용한 포먼트 분포(남성)

Figure 4. Formant distribution by qtone_script (male)

\subsection{2. 여성의 스크립트별 포먼트 분포}

그림 5는 fixed_5000n5_script를 이용한 여성의 포먼트 분포 다. 다른 모음에 비해 전설 모음/이, 에/의 포먼트 편차가 크게 나타난 것을 확인할 수 있다. 두 모음의 편차 범위가 $x$ 축 범위로 큰 것은 $\mathrm{F} 2$ 의 편차가 큰 것을 의미한다.

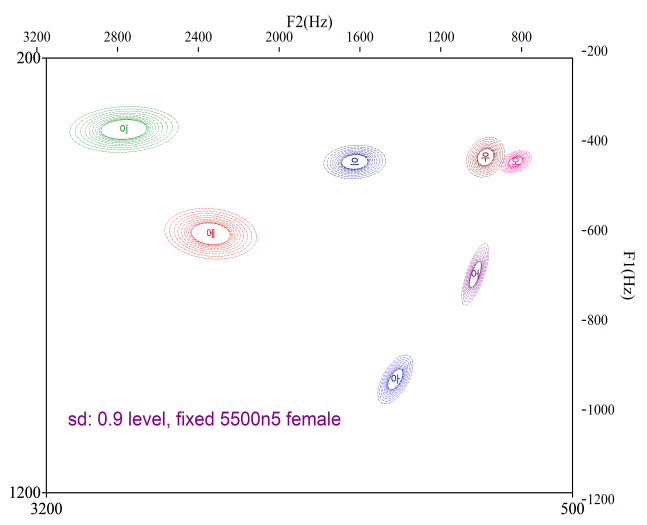

그림 5. fixed 5000n5 script를 이용한 포먼트 분포(여성)

Figure 5. Formant distribution by fixed_5000n5_script (female)

그림 6은 fixed_script를 이용한 여성의 포먼트 분포로 모음 / 오/를 제외하고 6 개 모음의 편차 범위가 큰 것을 알 수 있다. 그 림 5와 비교했을 때 특히 /우, 어/의 편차 범위가 크게 증가했고, /이, 에, 아/의 편차 범위도 증가한 것을 확인할 수 있다. 또한/우 /의 편차 범위가 증가하면서 영역이/오/와 일부 중복되는 것을 알수 있다.

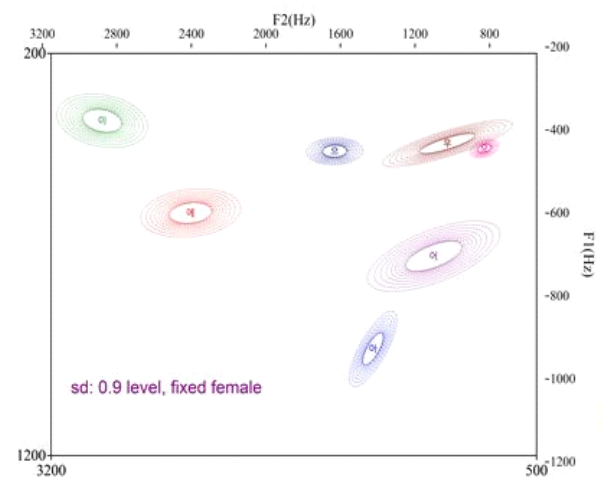

그림 6. fixed_script를 이용한 포먼트 분포(여성)

Figure 6. Formant distribution by fixed_script (female)

그림 7과 그림 8은 각각 linear_script, qtone_script를 이용한 포 먼트 분포로 서로 유사한 형태를 보인다. 7 개 모음의 각 영역이 독립적으로 구분되며 편차의 폭이 확연히 감소한 것을 확인할 수 있다. 특히 linear script의 포먼트 분포에서 모음/오, 우/의 영 역이 뚜렷하게 분리되는 것을 볼 수 있다. 


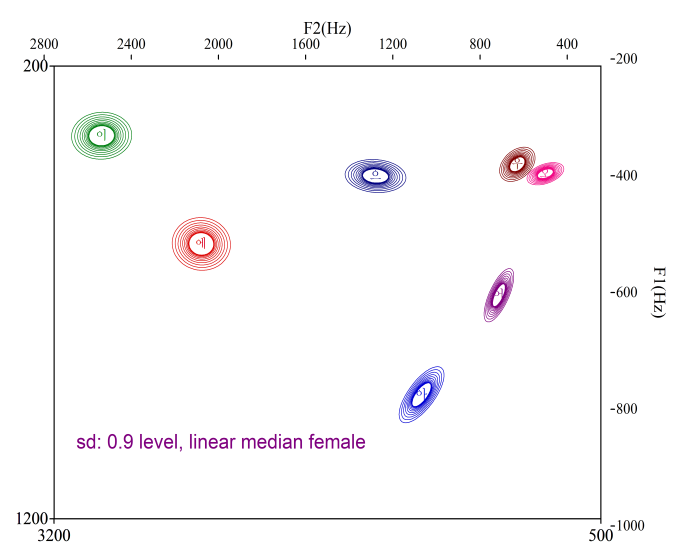

그림 7. Linear_script를 이용한 포먼트 분포(여성)

Figure 7. Formant distribution by linear_script (female)

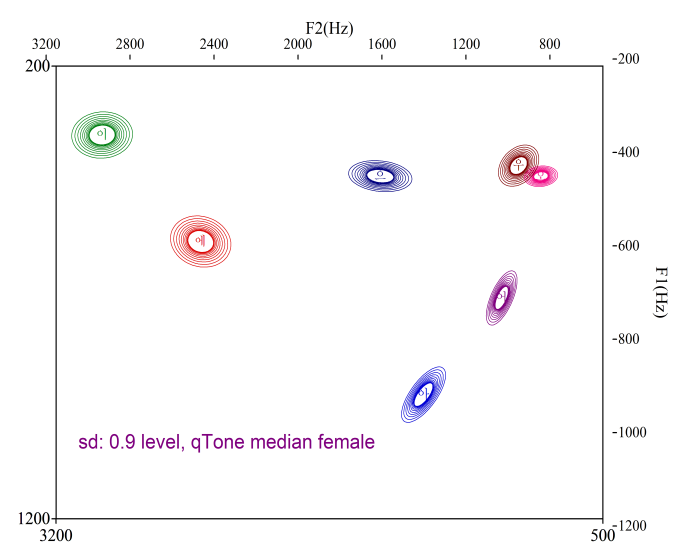

그림 8. qtone_script를 이용한 포먼트 분포(여성)

Figure 8. Formant distribution by qtone_script (female)

3.3. 모음별 포먼트 측정 결과 비교

\subsection{1. 포먼트 측정치에 대한 기술통계}

Fixed_5000n5_script, fixed_script, linear_script, qtone_script를 각각 이용하여 측정한 모음별 $\mathrm{F} 1, \mathrm{~F} 2$ 의 평균과 표준편차, 최대-

표 6. 각 스크립트별 모음별 포먼트값 기술통계(남성)

Table 6. Descriptive statistics for the formants according to each script for 7 vowels (male)

\begin{tabular}{|c|c|c|c|c|c|c|c|c|c|c|c|c|c|c|}
\hline \multirow{2}{*}{\multicolumn{2}{|c|}{ 모음(포먼트) }} & \multirow{2}{*}{$\mathrm{N}$} & \multicolumn{3}{|c|}{ Fixed_5000n5_script } & \multicolumn{3}{|c|}{ Fixed_script } & \multicolumn{3}{|c|}{ Linear_script } & \multicolumn{3}{|c|}{ Qtone script } \\
\hline & & & Mean & SD & Range & Mean & SD & Range & Mean & $\mathrm{SD}$ & Range & Mean & SD & Range \\
\hline \multirow{2}{*}{ 아 } & $\mathrm{F} 1(\mathrm{~Hz})$ & 16 & 739.9 & 67.6 & 196.2 & 762.6 & 67.8 & 192.8 & 740.6 & 60.7 & 208.9 & 749.1 & 61.9 & 194.2 \\
\hline & $\mathrm{F} 2(\mathrm{~Hz})$ & 16 & $1,188.2$ & 78.7 & 243.3 & $1,206.6$ & 82.7 & 254.3 & $1,159.2$ & 130.8 & 399.6 & $1,172.5$ & 119.2 & 414.2 \\
\hline \multirow{2}{*}{ 에 } & $\mathrm{F} 1(\mathrm{~Hz})$ & 16 & 453.0 & 48.3 & 170.5 & 460.1 & 46.6 & 157.2 & 455.4 & 47.8 & 165.9 & 455.0 & 48.1 & 67.6 \\
\hline & $\mathrm{F} 2(\mathrm{~Hz})$ & 16 & $1,980.4$ & 147.8 & 530.1 & $1,982.6$ & 140.9 & 494.0 & $1,987.4$ & 138.5 & 502.8 & $1,988.9$ & 142.2 & 510.0 \\
\hline \multirow{2}{*}{ 이 } & $\mathrm{F} 1(\mathrm{~Hz})$ & 16 & 279.4 & 28.0 & 86.6 & 283.3 & 334 & 116.9 & 279.8 & 28.3 & 86.3 & 283.2 & 30.7 & 04.4 \\
\hline & $\mathrm{F} 2(\mathrm{~Hz})$ & 16 & $2,299.9$ & 153.4 & 538.9 & $2,303.4$ & 155.3 & 612.6 & $2,294.0$ & 148.0 & 535.6 & $2,274.8$ & 143.3 & 00.9 \\
\hline \multirow{2}{*}{ 오 } & $\mathrm{F} 1(\mathrm{~Hz})$ & 16 & 376.1 & 40.9 & 161.7 & 365.7 & 29.1 & 98.9 & 370.1 & 35.5 & 109.8 & 372.5 & 34.8 & 103.0 \\
\hline & $\mathrm{F} 2(\mathrm{~Hz})$ & 16 & 679.0 & 79.4 & 297.4 & 679.4 & 72.8 & 253.2 & 697.9 & 94.6 & 322.7 & 702.1 & 86.2 & 290.4 \\
\hline \multirow{2}{*}{ 우 } & $\mathrm{F} 1(\mathrm{~Hz})$ & 16 & 366.6 & 91.7 & 381.5 & 326.9 & 22. & 66.0 & 330.3 & 30.2 & 98.0 & 331.1 & 25.0 & 75.2 \\
\hline & $\mathrm{F} 2(\mathrm{~Hz})$ & 16 & 980.5 & 421.0 & $1,626.7$ & 824.1 & 54.8 & 181.7 & 813.9 & 67.0 & 240.3 & 831.6 & 62.5 & 254.8 \\
\hline \multirow{2}{*}{ 으 } & F1(Hz) & 16 & 354.3 & 34.3 & 125.7 & 356.6 & 36.1 & 124.5 & 362.0 & 36.9 & 145.0 & 361.1 & 36.2 & 145.1 \\
\hline & $\mathrm{F} 2(\mathrm{~Hz})$ & 16 &, 411.2 & 122.9 & 434.9 & $1,403.5$ & 135.1 & 502.2 & $1,356.4$ & 176.5 & 580.6 & $1,346.3$ & 192.4 & 692.4 \\
\hline \multirow{2}{*}{ 어 } & F1(Hz) & 16 & 523.1 & 57.1 & 242.5 & 518.1 & 55.7 & 247.2 & 547.7 & 36.6 & 113.6 & 547.4 & 36.0 & 112.6 \\
\hline & $\mathrm{F} 2(\mathrm{~Hz})$ & 16 & 893.6 & 38.2 & 140.2 & 896.8 & 38.5 & 145.3 & 905.4 & 41.7 & 148.1 & 905.7 & 41.4 & 147.0 \\
\hline
\end{tabular}


표 7. 각 스크립트별 모음별 포먼트값 기술통계(여성)

Table 7. Descriptive statistics for the formants according to each script for 7 vowels (female)

\begin{tabular}{|c|c|c|c|c|c|c|c|c|c|c|c|c|c|c|}
\hline \multirow{2}{*}{\multicolumn{2}{|c|}{ 모음(포먼트) }} & \multirow{3}{*}{$\begin{array}{l}\mathrm{N} \\
28\end{array}$} & \multicolumn{3}{|c|}{ Fixed_5000n5_script } & \multicolumn{3}{|c|}{ Fixed_script } & \multicolumn{3}{|c|}{ Linear_script } & \multicolumn{3}{|c|}{ Qtone script } \\
\hline & & & Mean & SD & Range & Mean & SD & Range & Mean & SD & Range & Mean & SD & Range \\
\hline \multirow{2}{*}{ 아 } & $\mathrm{F} 1(\mathrm{~Hz})$ & & 939.0 & 59.2 & 213.4 & 934.8 & 98.5 & 466.2 & 925.9 & 65.2 & 304.4 & 925.7 & 64.5 & 278.5 \\
\hline & $\mathrm{F} 2(\mathrm{~Hz})$ & 28 & $1,409.0$ & 94.6 & 433.6 & $1,409.5$ & 139.9 & 605.3 & $1,385.3$ & 116.8 & 450.3 & $1,385.6$ & 114.5 & 453.1 \\
\hline \multirow{2}{*}{ 에 } & $\mathrm{F} 1(\mathrm{~Hz})$ & 28 & 604.1 & 61.4 & 212.6 & 596.7 & 63.4 & 202.0 & 592.7 & 61.2 & 205.2 & 587.4 & 59.5 & 184.8 \\
\hline & $\mathrm{F} 2(\mathrm{~Hz})$ & 28 & $2,356.5$ & 248.2 & $1,096.5$ & $2,424.0$ & 291.8 & $1,653.3$ & $2,473.0$ & 151.4 & 582.9 & $2,485.9$ & 157.0 & 14.8 \\
\hline \multirow{2}{*}{ 이 } & F1(H & 28 & 364.1 & 55.6 & 191.0 & 367.3 & 70.2 & 321.1 & 353.7 & 54.6 & 2.8 & 352.1 & 53.9 & 188.3 \\
\hline & $\mathrm{Hz}$ & 28 & ,801.8 & 93.6 & 5.1 & $2,915.2$ & 267.1 & 518.2 & $2,965.3$ & 156.4 & 45.9 & $2,972.6$ & 158.1 & 7.0 \\
\hline \multirow{2}{*}{ 오 } & $\mathrm{F}$ & 2 & 438. & 26 & 9 & 436.6 & 24.6 & 104.3 & 437.7 & 25.9 & 107.7 & 443.2 & 23.9 & .1 \\
\hline & $\mathrm{F} 2(\mathrm{~Hz})$ & 28 & 791.6 & 85 & 8 & 790.0 & 85.8 & 421.4 & 773.8 & 95.6 & 04.2 & 805.7 & 7.2 & 62.2 \\
\hline \multirow{2}{*}{ 우 } & $\mathrm{F} 1(\mathrm{~Hz})$ & 28 & 428.0 & 48.3 & 249.9 & 424.0 & 60.9 & 359.6 & 416.8 & 39.8 & 169.7 & 419.7 & 47.9 & 266.3 \\
\hline & $\mathrm{F} 2(\mathrm{~Hz})$ & 28 & 945.4 & 103.3 & & 996.1 & 378.7 & $2,135.8$ & 912.4 & 92. & 396.7 & 916.0 & 105.1 & 420.7 \\
\hline \multirow{2}{*}{ 으 } & & 28 & 438.9 & & & 443.0 & 35.4 & 140.1 & 442.7 & 38.4 & & 443.0 & 36.2 & 50.4 \\
\hline & $\mathrm{F} 2(\mathrm{~Hz})$ & 28 & $1,617.3$ & 167.5 & 99.3 & $1,622.8$ & 164.8 & 544.3 & ,612.7 & 158.1 & 549.0 & $1,599.2$ & 164.7 & 582.3 \\
\hline \multirow{2}{*}{ 어 } & $\mathrm{F} 1(\mathrm{~Hz})$ & 28 & 697.9 & 74.4 & 306.5 & 704.2 & 92.2 & 369.8 & 706.1 & 62.1 & 239.5 & 712.1 & 63.1 & 232.5 \\
\hline & F2(Hz) & 28 & 998.5 & 73.3 & 322.9 & $1,072.0$ & 388.7 & $2,155.6$ & $1,003.3$ & 77.2 & 331.1 & 999.5 & 80.1 & 343.6 \\
\hline
\end{tabular}

\subsection{2. 포먼트 측정치에 대한 반복측정 분산분석}

4 가지 스크립트의 포먼트 측정치에 대해 개체 내 변수는 4종 류 스크립트, 개체 간 변수는 7 모음으로 하여 혼합 반복측정 분 산분석(mixed repeated measure ANOVA)을 실시하였다. 남성의 경우 $\mathrm{F} 1$ 측정치는 스크립트 간 통계적으로 유의한 차이가 없는 것으로 나타났지만 $(p=824)$ 모음과의 상호작용 $(\mathrm{F} 1 \times$ 모음 $)$ 에서 유의한 차이가 있는 것으로 나타났다 $[F(7.641,133.714)=3.516$, $p<.01] . \mathrm{F} 2$ 측정치는 스크립트 간 통계적으로 유의한 차이가 없 는 것으로 나타났으며 $(p=.610)$, 모음과의 상호작용에서도 유의 한 차이가 없는 것으로 나타났다 $(p=.144)$.

4 개의 스크립트가 7 개 모음에 대해 측정한 포먼트에 어떠한 영향을 미치는지 세부적으로 살펴보기 위해 Bonferroni 조정한 사후검정을 실시하였을 때, 표 8에서 $\mathrm{F} 1, \mathrm{~F} 2$ 가 모음/우/의 경우, 스크립트에 따라 다르게 측정된 것을 확인할 수 있다. F1은 모 음 /우/에서 fixed_5000n5_script와 fixed_script $(p<.001)$, linear $\operatorname{script}(p<.01)$, qtone_script $(p<.01)$ 모든 쌍이 통계적으로 유의한 차이가 있는 것을 확인할 수 있다. F2의 경우, 모음 /우에서 fixed_5000n5_script와 fixed_script 간의 유의한 차이가 있는 것 으로 나타났다 $(p<.01)$.

표 8. 모음에 따른 스크립트별 F1 및 F2에 대한 짝대응비교(남성) Table 8. Pairwise comparisons for F1 and F2 for 2 vowels with respect to the 4 kinds of scripts (male)

\begin{tabular}{c|c|c|c|c|c}
\hline 변수 & 모음 & $\mathrm{I}$ & $\mathrm{J}$ & $\begin{array}{c}\text { 평균차이 } \\
(\mathrm{I}-\mathrm{J})\end{array}$ & $\mathrm{Sig}$. \\
\hline \multirow{2}{*}{$\begin{array}{c}\mathrm{F} 1 \\
(\mathrm{~Hz})\end{array}$} & \multirow{2}{*}{ 우 } & \multirow{2}{*}{ Fixed_5000n5 } & Fixed & 39.714 & $0.000^{* *}$ \\
\cline { 4 - 6 } & & Linear & 42.187 & $0.001^{* *}$ \\
\cline { 3 - 6 } $\begin{array}{c}\mathrm{F} 2 \\
(\mathrm{~Hz})\end{array}$ & 우 & Fixed_5000n5 & Fixed & 42.119 & $0.001^{* *}$ \\
\hline $\begin{array}{l}{ }^{* *} p<0.01 \\
p<0.390^{*}\end{array}$ & $0.001^{* *}$ \\
\hline
\end{tabular}

여성의 경우 $\mathrm{F} 1$ 측정치에 대해서 스크립트 간 유의한 차이가 있는 것으로 나타났고 $[F(1.897,358.475)=4.954, p<.001], \mathrm{F} 2$ 에서 도 스크립트 간 통계적으로 유의한 차이가 있는 것으로 나타났
다 $[F(1.856,350.863)=3.542, p<.05]$.

사후검정 결과, $\mathrm{F} 1$ 은 모음 / 아/에서 fixed_5000n5_script와 linear_script $(p<.05)$, qtone_script $(p<.001)$ 쌍에서 각각 유의한 차 이가 있는 것으로 나타났다(표 9). 또한 fixed_script와 linear_ $\operatorname{script}(p<.01)$, qtone_script $(p<.001)$ 쌍에서도 각각 유의한 차이가 있으며, linear_script와 qtone_sriprt 간 차이도 확인할 수 있다 $(p<.001) . \mathrm{F} 2$ 는 모음 /에/에서 fixed_script와 linear_script $(p<.05)$, 모음/이/에서 fixed_script와 linear_script 간 유의한 차이가 있는 것을 확인할 수 있다 $(p<.01)$.

표 9. 모음에 따른 스크립트별 F1 및 F2에 대한 짝대응비교(여성) Table 9. Pairwise comparisons for F1 and F2 for 3 vowels with respect to the 4 kinds of scripts (female)

\begin{tabular}{|c|c|c|c|c|c|}
\hline 변수 & 모음 & I & $\mathrm{J}$ & $\begin{array}{c}\text { 평균차이 } \\
\text { (I-J) }\end{array}$ & Sig. \\
\hline \multirow{5}{*}{$\begin{array}{c}\mathrm{F} 1 \\
(\mathrm{~Hz})\end{array}$} & \multirow{5}{*}{ 아 } & \multirow{2}{*}{ Fixed_5000n5 } & Linear & 31.971 & $0.017^{*}$ \\
\hline & & & Qtone & 46.825 & $0.000^{* *}$ \\
\hline & & \multirow{2}{*}{ Fixed } & Linear & 27.74 & $0.006^{* * *}$ \\
\hline & & & Qtone & 42.593 & $0.000^{* * *}$ \\
\hline & & Linear & Qtone & 14.853 & $0.038^{*}$ \\
\hline \multirow{2}{*}{$\begin{array}{c}\mathrm{F} 2 \\
(\mathrm{~Hz})\end{array}$} & 에 & Fixed & Linear & 229.945 & $0.011^{*}$ \\
\hline & 이 & Fixed & Linear & 264.144 & $0.002^{* *}$ \\
\hline
\end{tabular}

${ }^{*} p<0.05,{ }^{* *} p<0.01$

\section{4. 결론 및 논의}

프랏을 이용하여 성인의 모음 포먼트 분석에 필요한 최적 세 팅 조건을 찾을 수 있도록 스크립트를 이용한 포먼트 분석 및 통계 검정 과정을 기술하였다. 프랏에서 포먼트 분석 시 사용하 는 Burg 알고리듬의 주요 변수인 최대 포먼트 및 포먼트 개수에 대한 설계에 따라4가지 스크립트를 제시하였다.

먼저 프랏에서 권고하는 최대 포먼트(남성: $5,000 \mathrm{~Hz}$, 여성 : $5,500 \mathrm{~Hz})$, 포먼트 개수(5) 가이드 라인을 따르는 기본형 스크립 트(fixed_5000n5_script), 두 주요 변수 중 최대 포먼트 설정을 고 정하고, 포먼트 개수에만 변이를 설정한 포먼트-고정형 스크립 
트(fixed_script), 포먼트 개수 변이와 동시에 최대 포먼트 범위를 $100 \mathrm{~Hz}$ 단위로 확장한 선형-확장 스크립트(linear_script) 그리고 청지각적 인지 특성을 고려한 4분음 스크립트(qtone_script)까 지 총 4가지 스크립트를 이용하여 검출된 포먼트의 정확성과 그 분포의 개연성을 포먼트 산점도를 통해 비교하였다.

남성의 경우, fixed_5000n5_script에서 얻은 포먼트 분포는 원 순 후설 고모음/우/가 적절한 측정이 되지 않았고(그림 1), 나머 지 스크립트에 비해 넓은 편차 범위를 보였다(표 6). fixed $\operatorname{script}($ 그림 2), linear_script(그림 3), qtone_script(그림 4)에서 얻 은 포먼트 분포를 확인해 보면 모든 모음의 영역이 명확히 분리 된 것을 알 수 있는데 특히 linear_script, qtone_script에서는 /우/ 뿐만 아니라 중설모음/어/의 편차 또한 안정된 것을 확인할 수 있다. 여성의 경우, 스크립트 설계에 따른 포먼트 분포의 차이 가 두드러진다. 여성의 fixed_5000n5_script에서 얻은 포먼트 분 포에서 전설 모음/에, 이/의 포먼트 편차 범위가 큰 것을 확인할 수 있으며(그림 5), fixed_script의 포먼트 분포에서/에, 이/는 물 론 모음/우, 어, 아/의 편차 범위 모두 넓어진 것을 확인할 수 있 다(그림 6). 이와 비교하여 linear_script(그림 7), qtone_script(그 림 8)에서 얻은 포먼트 분포를 통해 모든 모음의 영역분리가 비 교적 명확하고, 포먼트 검출에 있어 안정적인 검출이 이루어졌 음을 좁은 편차 범위로 알 수 있다(표 7).

남녀의 경우를 종합해보면, 결국 최대 포먼트와 포먼트 개수 를 프랏의 권고 세팅만 이용하여 포먼트를 측정한 경우보다 변 이를 설정한 linear_script, qtone_script에서 더 안정적으로 포먼 트를 검출할 수 있음을 확인할 수 있다. 이를 통해 포먼트 분석 에서 사용하는 변수에 대한 적절한 설계가 필요하다는 결론을 얻게 된다.

스크립트마다 최적의 세팅으로 설정된 최대 포먼트, 포먼트 개수, 그 결과로 검출된 $\mathrm{F} 1, \mathrm{~F} 2$ 에 대해서 차이가 있는지 혼합 2 웨이 반복측정 분산분석으로 확인하였다. 최대 포먼트, 포먼트 개수의 세팅에서 각 스크립트 간 유의한 차이가 있는 것은 물론 모음 종류에 따라서도 두 세팅 변수가 스크립트 별로 달리 설정 되는 것을 확인할 수 있었다. 스크립트를 이용하여 측정한 F1, $\mathrm{F} 2$ 가 남성의 경우 원순 후설 고모음/우/에서 스크립트에 따라 달리 측정되는 것을 확인하였다. 여성의 경우에 F1은 모음 /아/ 에서, 전설 모음/에, 이/에서 스크립트에 따라 측정치가 달라지 는 것으로 나타났다. 이는 F1, F2 포먼트 검출이 분석 변수 설정 에 따라 달리 이루어지며, 포먼트 분석 시 주요한 두 변수인 최 대 포먼트, 포먼트 개수 설정이 모음과 성별의 특성을 반영해야 한다는 결론으로 이어진다.

다수의 선행연구는 F1과 F2를 이용한 포먼트 분석이 모음을 객관적으로 평가할 수 있다는 데에 동의한다(Park, 2008; Song \& Seong, 2018; Sim et al., 2016). 정확하게 측정된 포먼트값은 임상에서 치료의 객관성을 확보할 수 있는 수단이 된다.

성도의 공명은 복합적인 시스템으로 이루어져 있기 때문에 성별, 연령 등 여러 가지 요인들을 고려하여 세팅 값을 달리 해 야만 포먼트를 보다 정밀하게 측정할 수 있다. 앞서 언급했던 연구결과를 토대로 남녀 모두 전설 모음/에, 이/에서는 최대 포
먼트 범위를 높게 그리고 포먼트 개수를 적게 설정하는 것이 최 적의 포먼트 세팅인 것으로 제안할 수 있다. 또한 후설 모음/오, 우/에서는 최대 포먼트 범위는 낮게, 포먼트 개수는 많게 설정 하는 것이 포먼트 측정에 있어 편차를 최소화하고 정확한 측정 치를 얻는다는 결론을 얻었다. 이는 앞선 선행연구들과도 비슷 한 맥락이다(Kim \& Seong, 2016; Kim, 2015; Yang, 2019). 표 10 에 성별과 모음에 따라 최적의 포먼트 값을 얻을 수 있게 설계 된 세팅값(median)을 제시했다. 이는 성인 포먼트 측정에서 이 논문이 주장하는 정도의 정밀한 세팅을 원하는 연구자들에게 도움이 될 것이다.

표 10. 성별에 따른 각 모음별 최적의 포먼트 세팅(중앙값) Table 10. Optimal formant settings for each vowel with respect to the sexual difference (median value)

\begin{tabular}{c|c|c|c|c|c}
\hline \multirow{2}{*}{ 성별(모음) } & \multicolumn{2}{|c|}{ 최대 포먼트 } & \multicolumn{2}{c}{ 포먼트 개수 } \\
\cline { 2 - 6 } & Linear & Qtone & Linear & Qtone \\
\hline \multirow{3}{*}{ 남성 } & 에/이 & 4,700 & 4,627 & 4.5 & 4.5 \\
\cline { 2 - 6 } & 아/으/어 & 4,300 & $4,302.5$ & 5.5 & 5.5 \\
\cline { 2 - 6 } & 오/우 & 4,200 & 4,365 & 5.5 & 5.5 \\
\hline \multirow{3}{*}{ 여성 } & 에/이 & 5,400 & $5,598.5$ & 4.5 & 4.5 \\
\cline { 2 - 6 } & 아/으/어 & 5,300 & 5,205 & 5 & 5 \\
\cline { 2 - 6 } & 오/우 & 4,400 & 4,911 & 5.5 & 5.5 \\
\hline
\end{tabular}

결론적으로 모음 연구에서 성인의 포먼트 측정 시, 성별은 물 론, 모음 특성에 따라 최대 포먼트, 포먼트 개수를 달리 설정하 여 포먼트 분석을 실시하는 것이 앞으로의 포먼트 연구에 대한 신뢰성을 높일 것으로 판단된다. Park \& Seong(2018)에서는 성 인보다 다양한 변인이 있는 아동 연구에서 포먼트 측정 오차를 줄이기 위해 개별적인 최적의 세팅이 필요함을 밝혔다. 아동만 큼 다양한 변인이 영향을 미치는 것은 아니지만 성인 역시 모음 특성에 따라 발생할 수 있는 포먼트 측정 오차에 대해 그동안 간과되었던 부분이 있었다고 생각된다. 이러한 관점에서 본 연 구는 프랏을 이용하여 성인 포먼트를 더욱 정밀하게 측정할 수 있는 방법론을 제시했다는데 의의가 있다고 볼 수 있다.

본 연구는 녹음 대상자를 대전·충청 지역 거주자로 제한하였 기 때문에 경상, 전라 등 다른 방언 사용자나 북한 이탈 주민 등 에 대해서도 현재의 세팅값이 잘 적용되는지 살펴볼 필요가 있 다. 또한 비전형적인 음성 특성을 보이는 마비말장애나 음성장 애 대상자들의 경우에도 개인적인 특성을 고려하는 본 연구의 포먼트 측정 방법이 잘 적용되는지 확인할 필요가 있다.

\section{감사의 글}

이 논문은 2018년도 충남대학교 학술연구비의 지원을 받아 수행되었습니다.

\section{References}

Bae, J. (2003). The pronunciation of Korean. Seoul: Samgyeong. Behrman, A. (2007). Speech and voice science. Oxford: Plural Pub. 
Boersma, P., \& Weenink, D. (2014). Praat: Doing phonetics by computer [Computer program]. Retrieved from http://www.praat.org

Chiders, D. G. (1978). Modern spectrum analysis (pp. 252-255). New York: IEEE Press.

Escudero, P., Boersma, P., Rauber, A. S., \& Bion, R. A. H. (2009). A cross-dialect acoustic description of vowels: Brazilian and European Portuguese. Journal of Acoustical Society of America, 126(3), 1379-1393.

Fry, D. B. (1982). The physics of speech. Cambridge: Cambridge University Press.

Jin, S. M. (2004). Introduction of acoustic analysis of voice. Korean Journal of Otolaryngology-Head and Neck Surgery, 47(10), 943949.

Kent, R. D., \& Read, C. (2002). The acoustic analysis of speech (2nd ed.). New York: Thomson Learning.

Kim, E. (2012). (A) Study on native Chinese speaker's acquisition of Korean vowels (Master's thesis). Chungnam National University, Daejeon, Korea.

Kim, J. (2015). Comparison of phonetic characteristics of vowel pronunciation by children who grew up in a multi-lingual (Chinese-Korean) environment (Master's thesis). Chungnam National University, Daejeon, Korea.

Kim, J., \& Seong, C. (2016). The change of vowel characteristics for the Dysarthric speech along with speaking style. Phonetics and Speech Sciences, 8(3), 51-59.

Lee, J. R. (2017). Feasibility of acoustic analysis with laryngoscopic examination at outpatient clinic (Master's thesis). Ulsan National University, Ulsan, Korea.

Park, J., \& Seong, C. (2018) The implementation of children's automated fromant setting by Praat scripting. Phonetics and Speech Sciences, 10(4), 1-10.

Park, S. (2008). A validity study of formant analysis of vowel errors of children: Focused on vowel /u/. Journal of Speech \& Hearing Disorders, 17(3), 117-131.

Press, W. H., Teukolsky, S. A., Vetterling, W. T., \& Flannery, B. P. (1992). Numerical recipes in C: The art of scientific computing. (2nd ed.). Cambridge: Cambridge University Press.

Sapir, S., Ramig, L. O., Spielman, J. L., \& Fox, C. (2010). Formant centralization ratio: A proposal for a new acoustic measure of dysarthric speech. Journal of Speech, Language, and Hearing Research, 53(1), 114-125.

Seong, C. J. (2005). A formant analysis of the Korean monophthongs of the college students speaking Chungnam dialect, Language, 43, 189-213.

Seong, C. J., Kwon, O. W., Lee, J. H., \& Gim, C. G. (2008). A tonal analysis of East-Southern Gyeongnam dialect using Q-tone perceptual sense grade. Hangeul, 279, 5-33.

Song, I., \& Seong, C. (2018). Characteristics of 2 to 4 year old Korean children's production of monophthongs and diphthongs. Phonetics and Speech Sciences, 10(1), 65-74.

Sim, H., Choi, C., \& Choi, S. (2016). Characteristics of vowel formants, vowel space, and speech intelligibility produced by children aged 3 6 years. Audiology and Speech Research, 12(4), 260-268.

Yang, B. (2019). A comparison of normalized formant trajectories of English vowels produced by American men and women. Phonetics and Speech Sciences, 11(1), 1-8.

Yoon, T. J., \& Kang, Y. (2014). Monophthong analysis on a largescale speech corpus of read-style Korean. Phonetics and Speech Sciences, 6(3), 139-145.

\section{- 박지연 (Jiyeon Park)}

충남대학교 언어병리학과 박사과정

대전 유성구 대학로 99

Tel: 042-821-6391

Email: bpn0525jy@gmail.com

관심분야: 말장애, 분절음 분석

- 성철재 (Cheoljea Seong) 교신저자

충남대학교 언어학과 교수

대전 유성구 대학로 99

Tel: 042-821-6395

Email: cjseong49@gmail.com

관심분야: 분절음 및 운율분석 
부록

Linear_script의 핵심 코드는 다음과 같다.

for num to 6

numOf $=4+($ num -1$) * 0.5$

if gender $\$=" 1 "$

if vowel\$="a"

call formantset 40005900 numOf

elsif vowel $\$=" \mathrm{e} "$

call formantset 40005900 numOf

elsif vowel\$="i"

call formantset 40005900 numOf

elsif vowel $\$=" \mathrm{O} "$

call formantset 35005400 numOf

elsif gender $\$=" 2 "$

if vowel\$="a"

call formantset 45006400 numOf

elsif vowel $\$=" \mathrm{e} "$

call formantset 45006400 numOf

elsif vowel $\$=" i "$

call formantset 45006400 numOf

elsif vowel $\$=" \mathrm{o} "$

call formantset 40005900 numOf

endif

procedure formantset range_1 range_2 numOf

for formant_max from range_1 to range_2

select Sound 'fileName\$'

noprogress To Formant (burg)... 0 numOf formant_max 0.0250

select Formant 'fileName\$'

Rename... formantObj

Down to Table... no yes 6 no 3 yes 3 yes

Rename... 'formant_max'

formant_max=formant_max +99

endfor

noprogress Create Table with column names... 'vowel\$'_SD_LIST

...num_row Formant_max set_numOf_formants F1_SD F2_SD

...sumSD

select Table 'vowel\$' SD_LIST

sum $\min =$ Get minimum... sumSD

fin_row=Search column... sumSD 'sum_min'

fin_formant $=$ Get value... fin_row Formant_max

fin_F1SD=Get value... fin_row F1_SD fin $\mathrm{F} 2 \mathrm{SD}=$ Get value... fin row F2 SD

fin $\_$sum $=$Get value... fin row sumSD

endfor 


\title{
성인 포먼트 측정에서의 최적 세팅 구현: Praat software와 관련하여*
}

\author{
박지연 ${ }^{1} \cdot$ 성철재 $^{2}$ \\ 1충남대학교 언어병리학과, ${ }^{2}$ 충남대학교 언어학과
}

\begin{abstract}
국문초록
한국인 성인을 대상으로 최적의 포먼트 분석이 가능하도록 자동화된 프랏 스크립트를 구현하였다. 최적의 포먼트 분석이란 프랏에서 포먼트 분석 시 설정하는 2가지 세팅 파라미터(최대 포먼트, 포먼트 개수)를 조합하여 측정된 제 1 , 제 2 포먼트의 편차합이 최소일 때를 가리킨다. 포먼트 분석의 신뢰성을 높이기 위해서는 성별이나 모음의 종 류에 따라 LPC 차수를 다르게 설정해야 하는데 프랏 매뉴얼에서는 최대 포먼트 설정 값으로 남성 $5,000 \mathrm{~Hz}$, 여성 $5,500 \mathrm{~Hz}$, 측정개수는 5 개를 권고한다. 그러나 이렇게 권고된 포먼트 세팅 설정이 한국어 모음에 대해서도 타당한 지 검증이 필요하다. 본 연구에서 구현한 4가지 스크립트를 적용한 결과, 각 모음별 포먼트 산점도로 확인하였을 때 특히 여성의 경우 스크립트에 따라 측정된 포먼트 변이의 폭이 두드러지는 차이를 보였다. 포먼트 산점도와 통 계 결과를 통해 linear_script와 qtone_script가 포먼트 측정에서 더 신뢰성이 높은 것을 알 수 있었다. Linear_script, qtone_script에서 최적의 세팅으로 설정된 최대 포먼트와 포먼트 개수의 데이터 경향성을 살펴보면, 전설 모음 [이, 에]의 경우 권고 설정보다 최대 포먼트 값은 높게, 포먼트 개수의 값은 적게 설정되었다. 반면 후설모음 [오, 우]의 경우, 권고 설정보다 최대 포먼트 값은 낮게, 포먼트 개수의 값은 많게 설정되는 것을 확인할 수 있었다.
\end{abstract}

핵심어: Praat 스크립트, 포먼트 분석, 한국어 단모음

\section{참고문헌}

김은경 (2012). 중국인의 한국어 모음 습득 연구, 충남대학교 석 사학위논문.

김지연, 성철재 (2016). 경도 마비말장애 환자의 발화 유형에 따 른 모음 특성 비교. 말소리와 음성과학, 8(3), 51-59.

김지은 (2015). 중국인 다문화 아동이 산출한 모음의 음향음성학 적 특성. 충남대학교 석사학위논문.

박지연, 성철재 (2018). Praat을 이용한 아동 포먼트 자동 세팅 스 크립트 구현. 말소리와 음성과학, 10(4), 1-10.

박성지 (2008). 아동 모음 오류의 포먼트 분석의 타당성에 관한 연

구 -/u/ 모음을 중심으로-. 언어치료연구, 17(3), 117-131.

배주채 (2003). 한국어의 발음. 서울: 삼경문화사.

성철재 (2005). 충남지역 대학생들의 한국어 단모음 포먼트 분석. 언어학, 43, 189-213.

성철재, 권오욱, 이지향, 김차균 (2008). Q-tone 청취 등급을 이 용한 경남 동남부 방언 성조 분석. 한글, 279, 5-33.

송인미, 성철재(2018). 만 2 4세 아동의 단모음과 이중모음의 산
출 특징. 말소리와 음성과학, $10(1), 65-74$.

심화영, 최철희, 최성희 (2016). 만 3 6세 아동의 모음 포만트, 모 음 공간 및 말 명료도 특성. Audiology and Speech Research, 12(4), 260-268.

윤태진, 강윤정 (2014). 한국어 대용량발화말뭉치의 단모음분석. 말소리와 음성과학, 6(3), 139-145.

이재령 (2017). 외래에서 후두내시경 검사와 함께 시행하는 음항 학적 분석의 유용성. 울산대학교 석사학위논문.

진성민 (2004). 음향 음성학적 검사의 개요. 대한이비인후과학회 지, 47(10), 943-949.

\footnotetext{
* 이 논문은 2019년 한국음성학회 봄 학술대회 발표논문을 수정, 보완한 것입니다.
} 